\title{
Typologie des systèmes de production camelins dans la province de Laâyoune, Maroc
}

\begin{abstract}
Mots-clés
Dromadaire - Camelus dromedarius Jeune animal - Risque - Mortalité Classification - Epidémiologie - Maroc.
\end{abstract}

\author{
J.F. Michel ${ }^{1}$ M. Bengoumi ${ }^{2}$ P. Bonnet ${ }^{1}$ \\ K. Hidane ${ }^{3}$ K. Zro ${ }^{2}$ B. Faye ${ }^{1}$
}

\begin{abstract}
Résumé
Dans la province de Laâyoune au M aroc, la mortalité des jeunes dromadaires avant sevrage était importante sans qu'aucune étiologie évidente n'ait été identifiée. Afin de disposer d'une base d'échantillonage pour choisir les troupeaux qui feront l'objet d'un suivi, une typologie des éleveurs et de leurs pratiques a été réalisée par enquête transversale rétrospective auprès des éleveurs de la province de Laâyoune. Au terme de l'enquête, 170 éleveurs ont été interrogés, soit 10 p. 100 des éleveurs recensés, représentant 12000 dromadaires, soit 44 p. 100 des dromadaires recensés dans la province de Laâyoune. L'analyse multivariée effectuée sur les données regroupées parthèmes a permis de déterminer 4 types d'organisation des éleveurs en fonction de la taille du troupeau, 4 types de stratégie de déplacement selon les conditions bioclimatiques et 3 types de pratique d'élevage. Ces trois typologies n'étaient pas corrélées. D'après la quantité d'informations obtenues, le nombre d'interlocuteurs et la variété des élevages rencontrés, la majorité des situations épidémiologiques semble avoir été abordée. La typologie des systèmes de production camelin dans la province de Laâyoune la plus pertinente était fondée sur l'organisation des hommes autour du troupeau. Mise en relation avec la mortalité des jeunes dromadaires avant sevrage, cette typologie a permis d'émettre des hypothèses de facteurs de risque, comme l'existence de toxiques alimentaires, les pratiques de traite et de sevrage, le niveau de présence du propriétaire, la délégation de la gestion du troupeau et le degré d'encadrement humain des jeunes dromadaires. De plus, cette clé de typologie, facilement identifiable sur le terrain, pourra servir de base d'échantillonnage pour des protocoles de suivis individuels ultérieurs qui permettront de préciser les différentes pratiques d'élevage encore mal déterminées et de confirmer les hypothèses de facteurs de risque de la mortalité des jeunes.
\end{abstract}

\section{INTRODUCTION}

Depuis la Marche verte de 1975, le développement et la mise en valeur des provinces sahariennes, ex Sahara occidental espagnol, ont été la priorité de la politique marocaine. Au cours des 20 dernières années, ces provinces ont connu un développement démographique et économique sans précédent. Ainsi, de 14000 habitants en 1975, la ville de Laâyoune en compte aujourd'hui

1. CIRAD-EMVT, Campus international de Baillarguet, BP 5035, 34032 Montpellier Cedex 1, France

2. IAV Hassan II, BP 6202, Rabat Universités, Maroc

3. DPA, Laâyoune, Maroc plus de 140 000. Le dromadaire (Camelus dromedarius), par ses capacités à valoriser des zones de parcours à faibles potentialités, permet l'existence d'une activité d'élevage, activité principale et traditionnelle des populations Sahraouies de la province de Laâyoune et des autres provinces sahariennes. Dans cet élevage extensif, les troupeaux unis ou plurispécifiques, dromadaires et petits ruminants, sont conduits sur parcours selon un mode nomade (5). Quelques ateliers bovins et camelins laitiers périurbains fonctionnant en zero grazing (3) ont vu le jour du fait de la politique de soutien du Ministère de l'Agriculture et de la Mise en valeur agricole, mais ils restent marginaux.

Base sociale des tribus Sahraouis, cet élevage a subi les conséquences de plus de 20 ans de conflit dans cette région et le nombre de dromadaires a fortement diminué (15). Suite à la stabilisation militaire et à la sécurisation de la zone, conscient de son rôle so- 
cial, économique et écologique, capital pour le développement de cette région (7), le gouvernement marocain a entrepris, à la fin des années 80 , une politique de soutien à l'élevage camelin. Outre des mesures d'aide concrètes et directes, un vaste programme de recherche en coopération a été engagé.

Dans les conditions actuelles, la mortalité des jeunes dromadaires avant le sevrage est une contrainte majeure au développement de cet élevage (3). En effet, la mortalité des jeunes dromadaires avant sevrage, notamment suite à des diarrhées, demeure considérable (de 10 à 50 p. 100, selon les troupeaux) sans qu'aucune étiologie évidente ne soit identifiée. Partant de l'hypothèse que cette mortalité s'exprime dans un contexte de milieu et de système d'élevage donnés, une investigation large, de type écopathologique (10), est apparue comme la méthodologie la mieux appropriée pour tenter de résoudre cet état de fait lourd de conséquences. Pour appliquer cette méthode, qui repose sur un suivi fin des animaux, il importe de disposer d'une base d'échantillonnage des troupeaux s'appuyant sur les systèmes de pratique d'élevage. Il est donc nécessaire de réaliser au préalable une typologie des éleveurs et de leurs pratiques, en relation avec la mortalité des jeunes par une pré-enquête à l'échelle du troupeau. En outre, cette enquête devrait également permettre d'évaluer la réceptivité et la motivation des éleveurs vis-à-vis des actions engagées.

L'enquête a été réalisée sur le terrain auprès d'éleveurs de dromadaires de la province de Laâyoune, durant les mois de juillet et août 1996, dans le cadre de la coopération entre l'unité de coordination pour l'élevage camelin du CIRAD-EMVT de Montpellier, et l'Institut agro vétérinaire Hassan II de Rabat.

\section{MATERIEL ET METHODES}

\section{Zone d'étude}

Les provinces sahariennes du Maroc, dont Laâyoune est la capitale, sont situées sous le $28^{\mathrm{e}}$ parallèle $\mathrm{N}$ et représentent presque la moitié de la superficie du territoire marocain (figure 1). Les précipitations, très variables, sont en général inférieures à $100 \mathrm{~mm}$ par an et ont lieu de novembre à mars. Les amplitudes thermiques sont faibles, la température varie de 10 à $30{ }^{\circ} \mathrm{C}$ (4). Ce climat, de type désert côtier, devient plus désertique vers l'Est avec la diminution de l'influence océanique, l'augmentation des amplitudes thermiques et la diminution des précipitations. Il ne permet qu'un faible potentiel végétatif.

Les parcours représentent 1100000 ha dans la province de Laâyoune et 34000000 ha pour l'ensemble des provinces sahariennes (13). Ce territoire est relativement plat et l'altitude ne dépasse pas $200 \mathrm{~m}$. Les meilleurs parcours sont représentés par des dépressions à sols profonds qui recueillent les eaux de ruissellement, parfois utilisées pour la culture pluviale du blé, et les lits d'oued à sols relativement profonds. La végétation pâturée est essentiellement composée de Rhus tripartita, Atriplex halimus, Acacia tortilis et Launea arborescens. Les plateaux, formation majoritaire, portent peu de végétation qui laisse les sols à nu. Enfin la zone côtière, à végétation halophile de Salsola sp. et Zygophyllum sp., constitue une zone de parcours non négligeable en fin de saison sèche par la richesse de ses végétaux en eau (2, 14). Les points d'eau, multipliés depuis 20 ans, restent mal répartis.

Dans la province de Laâyoune, 27000 dromadaires ont été recensés (13), ce qui représentait 28 p. 100 du cheptel camelin du Maroc. Les troupeaux étaient de taille variable, de quelques dromadaires à quelques centaines, voire quelques milliers. Chaque troupeau appartenait à un ou plusieurs propriétaires qui pouvaient

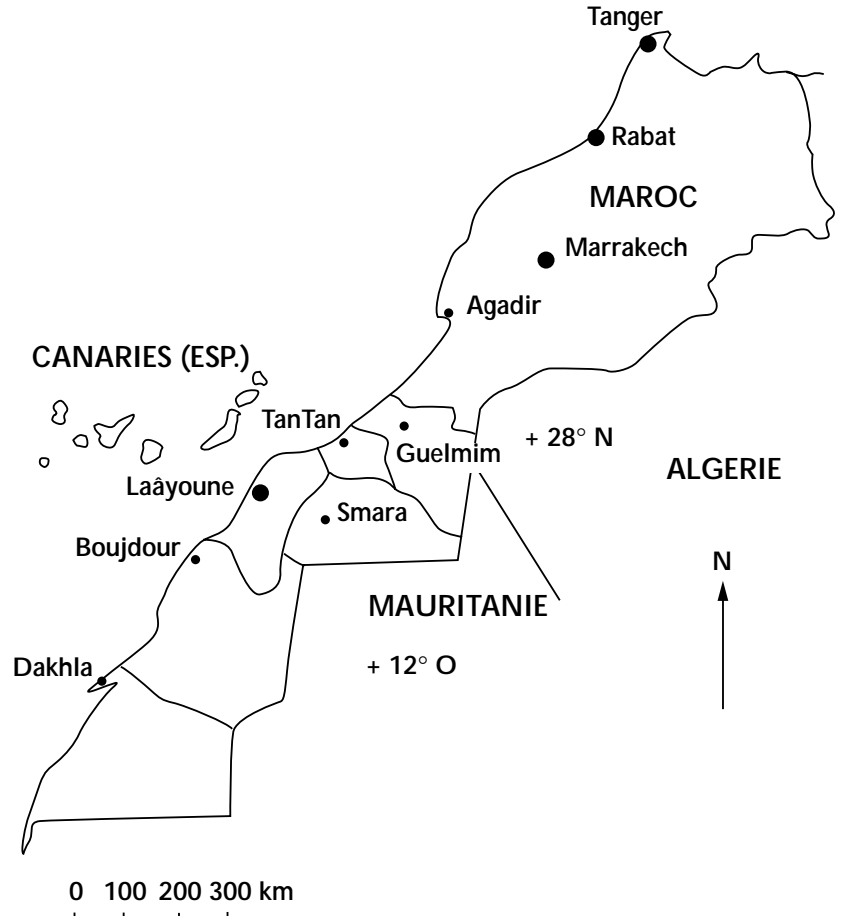

Figure 1 : province de Laâyoune et les provinces sahariennes au Maroc.

employer, selon la taille de leur troupeau, un ou plusieurs bergers (ouvrier exécutant les tâches quotidiennes) et un ou plusieurs contremaîtres, intermédiaires entre le propriétaire et le berger chargés de la gestion du troupeau.

\section{Questionnaire et modalités d'enquête}

Les données analysées proviennent d'une enquête transversale rétrospective. L'unité d'étude, le troupeau, a été définie comme un groupe de dromadaires présentant divers statuts physiologiques, conduits ensemble ou séparément, même de manière permanente, mais séparés par de faibles distances et soumis au même régime décisionnel humain. Chaque questionnaire, unité d'enquête, correspondait aux informations concernant un troupeau, unité d'étude.

Tous les représentants de troupeaux rencontrés ont été interrogés, auprès de leurs animaux ou, de manière anecdotique, en ville, selon leur capacité à répondre aux questions posées et la pertinence de leurs réponses. Ne disposant d'aucune base d'échantillonnage, cette procédure croisant les informations de diverses origines, a permis de décrire au mieux la variabilité des situations épidémiologiques.

Le questionnaire d'enquête a abordé tous les sujets touchant au troupeau. Il était divisé en deux parties : l'une concernait les hommes, propriétaire, berger, contremaître, et leur organisation autour du troupeau ; l'autre concernait le troupeau, les pratiques d'élevage et les événements survenus au cours des trois dernières années. Les questions étaient réparties sous forme de fiches, chaque fiche ayant trait à un type d'information. Les questions étaient pour la plupart des questions ouvertes, du fait du manque de références bibliographiques mais également pour éviter d'orienter les réponses des éleveurs. Après une courte pré-enquête et quelques modifications, le questionnaire définitif a été établi et utilisé lors des cinq semaines de l'enquête. 


\section{Stratégie d'analyse}

Les informations obtenues au cours de l'enquête ont été saisies sur le logiciel EPI INFO (7). Après recodage (redéfinition de classes, discrétisation des variables quantitatives), suppression des variables redondantes ou inexploitables et création de nouvelles variables, l'analyse des données a été effectuée. La typologie des systèmes d'élevage camelins s'est appuyée sur les analyses multivariées de l'école française des statistiques (analyse factorielle et classification) (11). Dans cette étude l'analyse multivariée a été réalisée sur le logiciel SPAD (17).

Afin de limiter les pertes d'information et l'émergence de variables très discriminantes mais peu pertinentes, notamment les variables de structure (9), les données ont été organisées en groupes homogènes, chacun se rapportant à un aspect particulier des systèmes de production. Quatre groupes ont été constitués (tableaux I à IV) :

- organisation humaine autour du troupeau ;

- structure du troupeau ;

- stratégies de déplacement adoptées par l'éleveur ;

- pratiques d'élevage proprement dites.

Une analyse des correspondances multiples (ACM) et une classification ascendante hiérarchique $(\mathrm{CAH})$ sur les facteurs obtenus par l'ACM ont été effectuées sur chacun de ces quatre groupes, permettant d'obtenir une typologie de chaque aspect des systèmes de production camelins. Ces quatre typologies ont ensuite été croisées pour tenter de déterminer, par un test du $\mathrm{chi}^{2}$, le degré de corrélation entre leurs classes respectives. La stratégie d'analyse est résumée dans la figure 2 .

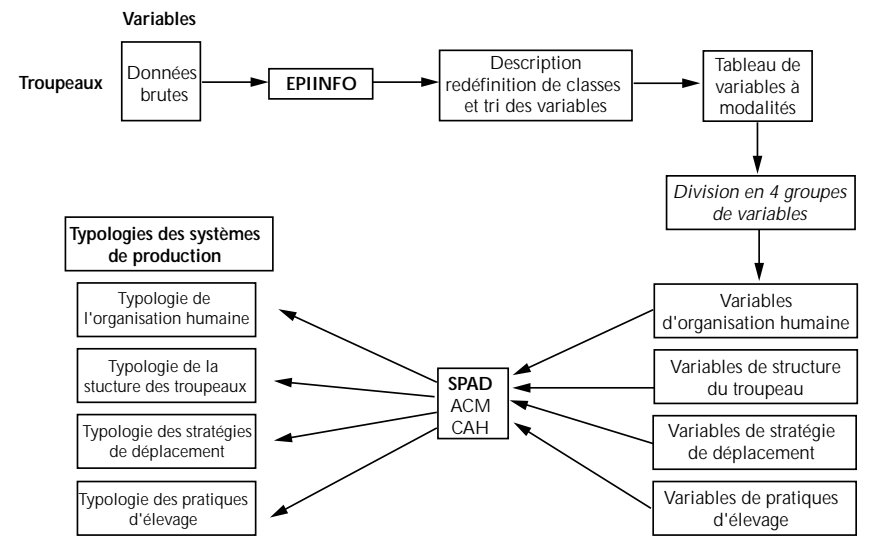

Figure 2 : schéma de la stratégie d'analyse.

RESU LTATS

Au terme de cette phase d'enquête, 170 éleveurs, soit 10 p. 100 des éleveurs recensés dans la province de Laâyoune, ont été interrogés. L'effectif de leur cheptel représentait 12000 dromadaires, soit 44 p. 100 des dromadaires recensés dans cette province.

\section{Typologie des systèmes de production sur la base de l'organisation des éleveurs}

Toutes les tribus citées dans la littérature (5) ont été représentées dans l'enquête. La tribu des Zerguiyines, avec 39 p. 100 des propriétaires interrogés, était majoritaire et sur-représentée par rapport à son importance réelle dans la province de Laâyoune, certaine-

Tableau I

Variable d'organisation humaine

\begin{tabular}{|c|c|c|c|}
\hline Code & Variable & Modalités & $\begin{array}{l}\text { Nb. de } \\
\text { troupeaux }\end{array}$ \\
\hline NOMBPROP & $\mathrm{Nb}$. de propriétaire(s) du troupeau & $\begin{array}{l}1: \text { Propriétaire seul } \\
2 \text { : Plusieurs propriétaires associés }\end{array}$ & $\begin{array}{r}155 \\
22\end{array}$ \\
\hline PRO PBERG & $\begin{array}{l}\text { Le propriétaire est aussi le berger } \\
\text { de son troupeau }\end{array}$ & $\begin{array}{l}1: \text { Oui } \\
2: \text { Non }\end{array}$ & $\begin{array}{r}51 \\
126\end{array}$ \\
\hline AUTMETPROP & Le propriétaire a un autre métier & $\begin{array}{l}1: \text { Oui } \\
2: \text { Non }\end{array}$ & $\begin{array}{r}46 \\
131\end{array}$ \\
\hline FRQ VISPRSS & $\begin{array}{l}\text { Fréquence des visites du propriétaire } \\
\text { au troupeau en saison sèche }\end{array}$ & $\begin{array}{l}1 \text { : En permanence ou tous les jours } \\
2 \text { : Au moins une fois par semaine } \\
3 \text { : Moins d'une fois par semaine, } \\
\text { mais plus d'une fois par mois } \\
4 \text { : Moins d'une fois par mois, } \\
\text { mais plus d'une fois par an }\end{array}$ & $\begin{array}{r}86 \\
58 \\
24 \\
9\end{array}$ \\
\hline FRQ VISPRSH & $\begin{array}{l}\text { Fréquence des visites du propriétaire } \\
\text { au troupeau en saison humide }\end{array}$ & Identiques à FRQ VISPRSS & ID \\
\hline NBCO N TREM & Nb. de contremaîtres employés & $\begin{array}{l}1: \text { Aucun } \\
2: 1 \text { ou plus }\end{array}$ & $\begin{array}{r}165 \\
12\end{array}$ \\
\hline NBBERG & $\mathrm{Nb}$. de bergers employés & $\begin{array}{l}1: \text { Aucun } \\
2: 1 \text { seul } \\
3: \text { Plus d'un }\end{array}$ & $\begin{array}{r}36 \\
121 \\
20\end{array}$ \\
\hline
\end{tabular}




\section{Tableau II}

Variables de stratégie de déplacement

\begin{tabular}{|c|c|c|c|}
\hline Code & Variable & Modalités & $\begin{array}{l}\text { Nb. de } \\
\text { troupeaux }\end{array}$ \\
\hline LOCTSS96 & $\begin{array}{l}\text { Localisation du troupeau } \\
\text { en saison sèche } 1996\end{array}$ & $\begin{array}{l}1: \text { Côte Nord } \\
2: \text { Côte Sud proche } \\
3: \text { Côte Sud éloignée } \\
4: \text { Est proche } \\
5: \text { Est éloigné } \\
6: \text { Sud proche } \\
7: \text { Sud éloigné }\end{array}$ & \\
\hline LOCTSH 95 & $\begin{array}{l}\text { Localisation du troupeau } \\
\text { en saison humide } 1995\end{array}$ & Identiques à LOCTSS96 & \\
\hline LOCTSS95 & $\begin{array}{l}\text { Localisation du troupeau } \\
\text { en saison sèche } 1995\end{array}$ & Identiques à LO CTSS96 & \\
\hline LOCTSH 94 & $\begin{array}{l}\text { Localisation du troupeau } \\
\text { en saison humide } 1994\end{array}$ & Identiques à LO CTSS96 & \\
\hline DEP96 & Déplacement du troupeau en 1996 & $\begin{array}{l}1: \text { Oui } \\
2: \text { Non }\end{array}$ & $\begin{array}{r}127 \\
50\end{array}$ \\
\hline DEP95 & Déplacement du troupeau en 1995 & $\begin{array}{l}1: \text { Oui } \\
2: \text { Non }\end{array}$ & $\begin{array}{l}98 \\
79\end{array}$ \\
\hline
\end{tabular}

Tableau III

Variables de structure du troupeau

\begin{tabular}{|c|c|c|c|}
\hline Code & Variable & Modalités & $\begin{array}{l}\text { Nb. de } \\
\text { troupeaux }\end{array}$ \\
\hline ESPPRESENT & Espèces présentes dans le troupeau & $\begin{array}{l}1 \text { : Dromadaires seuls } \\
2 \text { : Dromadaires et petits ruminants }\end{array}$ & $\begin{array}{l}88 \\
89\end{array}$ \\
\hline EFFECTIF & $\begin{array}{l}\mathrm{Nb} \text {. d'animaux dans le troupeau } \\
\text { de dromadaires }\end{array}$ & $\begin{array}{l}1: \text { Moins de } 50 \\
2: \text { Entre } 50 \text { et } 100 \\
3: \text { Entre } 100 \text { et } 300 \\
4: \text { Plus de } 300\end{array}$ & $\begin{array}{r}84 \\
50 \\
36 \\
7\end{array}$ \\
\hline NBFEM REPRO & $\mathrm{Nb}$. de femelles en âge de reproduire & $\begin{array}{l}1: \text { Moins de } 20 \text { femelles } \\
2: \text { Entre } 20 \text { et } 50 \text { femelles } \\
3: \text { Entre } 50 \text { et } 100 \text { femelles } \\
4: \text { Plus de } 100 \text { femelles }\end{array}$ & $\begin{array}{l}60 \\
64 \\
35 \\
18\end{array}$ \\
\hline ALLO TEM EN T & Pratiques de mise en lot & $\begin{array}{l}1: \text { ui } \\
2: \text { Non }\end{array}$ & $\begin{array}{r}56 \\
121\end{array}$ \\
\hline NBFEMTRTES & $\mathrm{Nb}$. de femelles traites & $\begin{array}{l}1: \text { Toutes } \\
2: \text { Une partie seulement }\end{array}$ & $\begin{array}{r}129 \\
48\end{array}$ \\
\hline
\end{tabular}

ment du fait de la présence d'un guide Zerguiyine. Quelle que fût leur tribu d'origine, les éleveurs avaient des rapports variés avec leur troupeau de dromadaires et s'organisaient de différentes manières pour le gérer. Les éleveurs restaient à des degrés divers auprès de leurs dromadaires sur les parcours. Quatre-vingt-quinze pour cent avaient un lieu d'habitation fixe en ville et la fréquence de leurs visites au troupeau était extrêmement variable. Pour la conduite journalière des dromadaires, 29 p. 100 des propriétaires étaient eux-mêmes les bergers, mais pouvaient employer en plus un ou plusieurs bergers, 69 p. 100 des propriétaires employaient un berger et 11 p. 100 des propriétaires employaient plus d'un berger. En revanche, ceux qui faisaient appel aux services d'un contremaître - terme usité localement - salarié étaient peu nombreux et représentaient 7 p. 100 des propriétaires interrogés, mais ce rôle pouvait être rempli par un fils ou un propriétaire associé et n'était alors pas compté parmi les contremaîtres. Le contremaître agissait comme intermédiaire entre le propriétaire et les bergers. Il représentait le propriétaire auprès du troupeau et avait un réel pouvoir décisionnel sur la conduite d'élevage, contrairement aux bergers. Les bergers, pour la plupart, avaient un statut temporaire, 65 p. 100 d'entre eux ne restaient pas plus de deux ans auprès du même troupeau. Leur âge variait entre 15 et 50 ans, 71 p. 100 étaient originaires des régions d'Ouarzazate et d'Essaouira situées plus au nord, et 29 p. 100 des provinces du sud. Dans 73 p. 100 des cas ils n'avaient aucun pouvoir décisionnel et seuls 27 p. 100 d'entre eux avaient un rôle consultatif auprès du propriétaire et pouvaient orienter ses décisions.

Les auteurs n'ont retenu que les trois premiers axes de l'analyse factorielle en composantes multiples (63 p. 100 de l'inertie du nuage). Les variables de fréquence des visites du propriétaire au troupeau en saison sèche et en saison humide (FRQVISPRSS et 
Tableau IV

Variables de pratiques d'élevage

\begin{tabular}{|c|c|c|c|}
\hline Code & Variable & Modalités & $\begin{array}{l}\mathrm{Nb} \text {. de } \\
\text { troupeaux }\end{array}$ \\
\hline \multirow[t]{4}{*}{ SURVMBAS } & \multirow[t]{4}{*}{ Surveillance des femelles qui vont mettre bas } & 1 : Toutes les femelles sont entravées & 51 \\
\hline & & 2 : Une partie des femelles est entravée & 34 \\
\hline & & 3 : Surveillance visuelle & 77 \\
\hline & & 4 : Aucune surveillance & 15 \\
\hline \multirow[t]{2}{*}{ COLOSTAVOL } & Prise de colostrum par un jeune vigoureux & $1:$ A volonté & 154 \\
\hline & né normalement & 2 : Limitée par l'éleveur & 23 \\
\hline \multirow[t]{2}{*}{ AIDECOL } & Pratiques de distribution du colostrum & $1:$ A volonté & 12 \\
\hline & aux nouveaux nés faibles & 2 : Limitée par l'éleveur & 165 \\
\hline \multirow[t]{4}{*}{ TRAITEM IN } & Temps entre la mise bas et le début & $1: 1$ à 2 mois & 24 \\
\hline & de la traite des femelles & $2: 3$ à 4 mois & 86 \\
\hline & & $3: 5$ à 6 mois & 60 \\
\hline & & 4 : Plus de 7 mois & 7 \\
\hline \multirow[t]{3}{*}{ SEVMIN } & Age minimum au sevrage & 1 : Moins d'un an & 25 \\
\hline & & $2: 1$ an & 137 \\
\hline & & $3:$ Plus d'un an & 15 \\
\hline \multirow[t]{3}{*}{ MODESEVRAGE } & Pratiques de sevrage & $\begin{array}{l}1 \text { : L'homme n'intervient que si la femelle } \\
\text { ne le fait pas spontanément }\end{array}$ & 70 \\
\hline & & $\begin{array}{l}2 \text { : L'homme sèvre systématiquement } \\
\text { lui-même les chamelons }\end{array}$ & 87 \\
\hline & & $\begin{array}{l}3 \text { : La femelle sèvre toujours } \\
\text { elle-même son chamelon }\end{array}$ & 20 \\
\hline \multirow[t]{2}{*}{ PLTESTO X } & Connaissance des plantes toxiques & $1:$ O ui & 74 \\
\hline & par l'interlocuteur & $2:$ Non & 103 \\
\hline \multirow[t]{2}{*}{ SU PPLEM } & Pratiques de supplémentation & $1:$ Au moins lors des années de sécheresse & 169 \\
\hline & des dromadaires & 2 : Jamais & 7 \\
\hline \multirow[t]{3}{*}{ TRAITEM ENT } & Pratiques thérapeutiques des éleveurs & $1:$ Modernes et traditionnelles & 135 \\
\hline & & 2 : Modernes seulement & 38 \\
\hline & & 3 : Traditionnelles seulement & 4 \\
\hline \multirow[t]{2}{*}{ DEPAREXT } & Déparasitage externe & $1: 0$ ui & 171 \\
\hline & & $2:$ Non & 6 \\
\hline \multirow[t]{2}{*}{ VACCIN } & Vaccination contre la variole cameline & $1: 0$ ui & 18 \\
\hline & & $2:$ Non & 159 \\
\hline
\end{tabular}

FRQVISPRSH) avaient les contributions aux axes les plus fortes. La classification ascendante hiérarchique effectuée sur les facteurs permet d'identifier quatre classes qui se dessinent en fonction des modalités des visites (tableau I). Le nombre de dromadaires par troupeau (EFFECTIF, tableau III), introduit comme variable illustrative, est fortement corrélé aux classes obtenues (tableau V).

Ces quatre classes (tableau V) peuvent être interprétées comme quatre types d'élevage :

- la première classe comprenait 87 petits éleveurs, souvent bergers, souvent associés, présents en permanence avec leur troupeau qui comptait généralement moins de 50 dromadaires, avec un minimum de personnel employé. L'élevage constituait pour ces éleveurs la seule ressource ;

- la classe 2 correspondait à 51 éleveurs de moyenne importance. Leur troupeau pouvait dépasser les 100 dromadaires. Ces éleveurs employaient le plus souvent un berger pour effectuer les tâches quotidiennes et avaient une autre activité professionnelle sans diminuer pour autant leur présence auprès de leur troupeau. L'élevage était pour eux une activité nécessaire, complétée par une activité annexe ;

- la classe 3 était celle des gros éleveurs $(\mathrm{n}=30)$, peu présents auprès de leur troupeau. Ils avaient d'autres activités que l'élevage et employaient du personnel pour s'occuper du troupeau. Souvent de gros commerçants ou des cadres, l'élevage camelin représentait pour eux une source de diversification de leur activité, mais surtout un moyen d'épargne ;

- la classe 4 correspondait aux très gros éleveurs $(n=9)$ qui possédaient de 300 à plus de 1000 dromadaires répartis en plusieurs troupeaux, confiés à la gestion de contremaitres. Pour ces éleveurs très rarement en contact avec leurs troupeaux, l'élevage représentait une source de prestige dans la société Sahraouie. C'était un moyen d'épargne mais également un secteur d'activité comme un autre, d'autant plus rentable que ces éleveurs naisseurs, dits « pépiniéristes », liés par contrat aux services de l'élevage, bénéficiaient de subventions lors de la vente de leurs produits.

\section{Typologie des systèmes de production sur la base de la structure des troupeaux}

La taille des troupeaux variait de 2 à 900 dromadaires, la moyenne était de 79 animaux par troupeau avec un écart-type de 106, mais la médiane était égale à 50 individus, traduisant l'influence sur la moyenne de quelques très gros troupeaux. En effet, 24 p. 100 des éleveurs interrogés possédaient 59 p. 100 des dromadaires (figure 3). Cinquante pour cent des troupeaux comptaient entre 20 et 95 (premier et troisième quartile) dromadaires. Les troupeaux 


\begin{tabular}{|c|c|c|c|c|c|}
\hline \multirow[t]{3}{*}{$\begin{array}{r}\text { Descr } \\
\text { de l'orga } \\
\text { des modal } \\
\text { Les va }\end{array}$} & \multirow[t]{3}{*}{$\begin{array}{l}\text { iption } \\
\text { nisatio } \\
\text { ités de } \\
\text { riables } \\
\text { aux v }\end{array}$} & $\begin{array}{l}\text { sses } \\
\text { aine } \\
\text { bles } \\
\text { ique } \\
\text { sill }\end{array}$ & $\begin{array}{l}\text { la ty } \\
\text { le p } \\
\text { is ch } \\
\text { orres } \\
\text { ative }\end{array}$ & $\begin{array}{l}\text { logie } \\
\text { rcent } \\
\text { ue cl } \\
\text { nden }\end{array}$ & \\
\hline & & \multicolumn{4}{|c|}{ Classes de la typologie } \\
\hline & & 1 & 2 & 3 & 4 \\
\hline \multicolumn{2}{|l|}{$\begin{array}{l}\text { Nb. de } \\
\text { troupeaux }\end{array}$} & 87 & 51 & 30 & 9 \\
\hline \multirow{5}{*}{ FRQ VISPRSS } & & \multicolumn{4}{|c|}{$\%$} \\
\hline & 1 & 92 & 6 & 10 & - \\
\hline & 2 & 8 & 94 & 10 & - \\
\hline & 3 & - & - & 80 & - \\
\hline & 4 & - & - & - & 100 \\
\hline \multirow[t]{2}{*}{ NBCO NTREM } & 1 & 99 & 98 & 90 & 22 \\
\hline & 2 & 1 & 2 & 10 & 78 \\
\hline \multirow[t]{3}{*}{ NBBERG } & 1 & 41 & - & - & - \\
\hline & 2 & 56 & 96 & 70 & 33 \\
\hline & 3 & 3 & 4 & 30 & 67 \\
\hline \multirow[t]{2}{*}{ PRO PBERG } & 1 & 57 & 2 & - & - \\
\hline & 2 & 43 & 98 & 100 & 100 \\
\hline \multirow[t]{2}{*}{ AUTMETPROP } & 1 & 10 & 40 & 33 & 78 \\
\hline & 2 & 90 & 60 & 67 & 22 \\
\hline \multirow[t]{2}{*}{ NOMBPROP } & 1 & 84 & 94 & 83 & 100 \\
\hline & 2 & 16 & 6 & 17 & 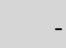 \\
\hline \multirow[t]{4}{*}{ EFFECTIF } & 1 & 60 & 43 & 30 & 11 \\
\hline & 2 & 25 & 33 & 37 & - \\
\hline & 3 & 14 & 24 & 27 & 44 \\
\hline & 4 & 1 & - & 6 & 45 \\
\hline \multirow[t]{2}{*}{ ESPPRESENT } & 1 & 40 & 50 & 67 & 67 \\
\hline & 2 & 60 & 50 & 33 & 33 \\
\hline
\end{tabular}

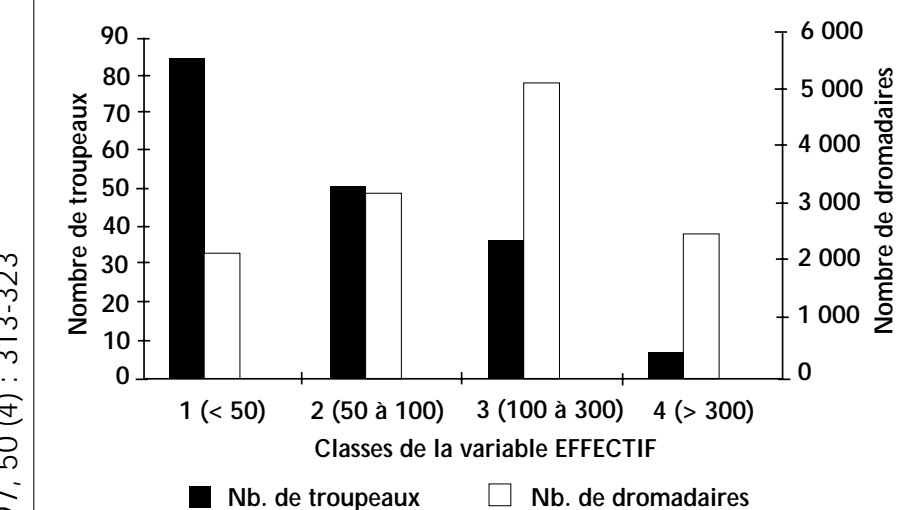

Figure 3 : répartition du nombre de dromadaires et du nombre de troupeaux dans chaque classe de la variable EFFECTIF.

étaient donc assez gros en terme d'effectif, ce qui représentait un capital important pour les éleveurs concernés (rappelons que le prix d'un dromadaire variait de 4000 à plus de $7000 \mathrm{FF}$ ).

Certaines variables étaient corrélées au nombre de dromadaires dans le troupeau, comme la présence de petits ruminants, la mise en lot, le pourcentage de femelles en lactation traites et les pratiques de supplémentation. La possession d'une pharmacie, ou ré- serve de produits vétérinaires, semblait également conditionnée par la taille du troupeau. Elle était plus fréquente dans les grands troupeaux. Les pratiques de supplémentation étaient assez homogènes. Des aliments étaient distribués aux animaux en saison sèche, mais seulement 5 p. 100 des éleveurs supplémentaient chaque année quelles qu'aient été les conditions alimentaires, alors que 95 p. 100 des éleveurs ne supplémentaient que si l'année était particulièrement sèche. Les aliments employés étaient essentiellement la paille, l'orge et la pulpe sèche de betterave. Certains éleveurs utilisaient aussi du foin de luzerne, du son, du pain, des aliments composés et tous les aliments qu'ils pouvaient se procurer au souk à moindre coût. Les animaux supplémentés en priorité étaient les femelles suitées, les géniteurs et les animaux faibles ou malades, les femelles gestantes n'étaient jamais supplémentées. Selon la taille du troupeau les éleveurs supplémentaient tous les animaux ou seulement les catégories précitées.

Parmi les variables actives analysées (tableau III), le nombre d'animaux (EFFECTIF), le nombre de femelles en âge de reproduire dans le troupeau (NBFEMREPRO), ainsi que la mise en lots des animaux (ALLOTEMENT), avaient les plus fortes contributions aux trois premiers axes déterminés par l'ACM (50 p. 100 de l'inertie du nuage). La classification permet d'identifier quatre classes caractérisées en premier lieu par le nombre de dromadaires dans le troupeau (tableau VI) :

- la classe des petits troupeaux de moins de 50 dromadaires $(n=84)$, conduits ensemble en permanence et avec d'autres espèces ;

- la classe des troupeaux moyens $(\mathrm{n}=50)$, de 50 à 100 dromadaires, rarement séparés en lots ;

- la classe des gros troupeaux $(\mathrm{n}=36)$, de 100 à 300 dromadaires, séparés en lots au moins en saison de reproduction et rarement conduits avec d'autres espèces ;

- la classe des très gros troupeaux de plus de 300 dromadaires $(n=7)$, divisés en permanence en lots. L'unité du troupeau restait conservée par la circulation des animaux d'un lot à l'autre, par les faibles distances qui les séparaient, et par la même stratégie d'élevage.

\section{Tableau VI}

Description des classes de la typologie de la structure des troupeaux par le pourcentage des modalités des variables dans chaque classe

\begin{tabular}{lrrrrr} 
& & \multicolumn{5}{c}{ Classes de la typologie } \\
\cline { 3 - 6 } Variable & Mod. & $\mathbf{1}$ & $\mathbf{2}$ & $\mathbf{3}$ & $\mathbf{4}$ \\
\hline $\begin{array}{l}\text { Nb. de } \\
\text { troupeaux }\end{array}$ & & $\mathbf{8 4}$ & $\mathbf{5 0}$ & $\mathbf{3 6}$ & $\mathbf{7}$ \\
& & & & & \\
EFFECTIF & 1 & 100 & - & - & - \\
& 2 & - & 100 & - & - \\
& 3 & - & - & 100 & - \\
ESPPRESENT & 1 & - & - & - & 100 \\
& 2 & 33 & 52 & 75 & 100 \\
NBFEM REPRO & 1 & 67 & 48 & 25 & - \\
& 2 & 65 & 10 & - & 14 \\
& 3 & 35 & 62 & 11 & - \\
ALLOTEMENT & 1 & - & 28 & 56 & - \\
& 4 & - & - & 33 & 86 \\
N BFEM TRTES & 2 & - & 25 & 95 & 100 \\
& 1 & 85 & 75 & 5 & - \\
& 2 & 15 & 20 & 44 & 75
\end{tabular}




\section{Typologie des systèmes de production basée sur la stratégie de déplacement}

Les éleveurs de dromadaires dans les provinces du sud étaient traditionnellement nomades, mais leurs déplacements différaient, selon les éleveurs et selon les années, et plusieurs stratégies semblaient se dessiner. Afin de préciser ces déplacements, des zones (aire de déplacement de $40 \mathrm{~km}$ de rayon), elles-mêmes situées dans des régions (ensemble géographique homogène), ont été définies. Les zones ont été déterminées pour chaque localisation de troupeau. Chaque zone représentait une aire de parcours fixe et les déplacements des animaux en son sein ne nécessitaient pas de déplacer le campement des bergers. Si le troupeau se déplaçait au delà de $40 \mathrm{~km}$, le campement était déplacé sur la nouvelle zone de parcours. Le déplacement, réel et important, était alors pris en compte. Les zones ont été rassemblées en régions homogènes qui servaient à localiser de façon grossière les troupeaux en saison sèche et en saison humide, et ainsi à déterminer leurs trajectoires au cours de l'année. Finalement, un troupeau se déplaçait s'il changeait de zone, mais il pouvait se déplacer à l'intérieur d'une même région. La période de déplacement des troupeaux correspondait à la saison humide. Le départ avait lieu au cours des mois d'octobre et de novembre, le retour en mars, avril ou mai, selon les années. En 1995, année de sécheresse, 45 p . 100 des troupeaux ne se sont pas déplacés. Ils sont restés dans une même zone, non loin d'un point d'eau, alors que 55 p. 100 des troupeaux se sont déplacés. En 1996, les conditions alimentaires ont été meilleures et 72 p. 100 des troupeaux se sont déplacés, alors que 28 p. 100 sont restés dans la même zone. Les troupeaux qui ne se sont pas déplacés en 1996 ne s'étaient pas non plus déplacés en 1995. Les trajectoires de déplacement ont varié selon les troupeaux et pour un même troupeau, selon les années, mais il semble que beaucoup de troupeaux revenaient toujours sur les mêmes parcours en saison sèche, du moins sur les trois années qui ont été explorées, puisque 70 p. 100 des troupeaux passaient la saison sèche au même endroit chaque année. En revanche les troupeaux changeaient de zone au cours d'une saison humide. Si le mode d'élevage était sédentaire en saison sèche il était donc parfaitement nomade pour les troupeaux qui se déplaçaient en saison humide, et conditionné par la localisation des parcours ayant reçu des précipitations.

Pour parvenir à une détermination des stratégies générales de déplacement, une typologie des déplacements a été réalisée pour chaque année, 1996 et 1995, leur synthèse représentant la typologie des stratégies générales de déplacement des éleveurs selon les conditions bioclimatiques, donc alimentaires.

L'ACM et la CAH effectuées sur les variables de déplacement en 1995 (LOCTSS95, LOCTSH94 et DEP95) (tableau II) ont déterminé trois classes, déséquilibrées, de troupeaux qui ont adopté des stratégies de déplacement différentes lors de cette année de sécheresse :

- la première classe représentait 95 troupeaux, dont la localisation en saison sèche était variable d'une année à l'autre, qui ont effectué un déplacement de plus de $500 \mathrm{~km}$ vers l'intérieur, au sud ou à l'est de Laâyoune ;

- la deuxième classe ne comptait que 13 troupeaux qui ne se sont pas déplacés mais qui étaient déjà, en saison sèche, situés sur des parcours à plus de $300 \mathrm{~km}$ de Laâyoune au sud ou à l'est. Ils n'ont pas eu à se déplacer pour trouver des parcours plus riches ;

- la troisième classe représentait 59 troupeaux qui ne se sont pas déplacés cette année-là et dont la localisation en saison sèche était toujours la même chaque année.
Ces trois classes représentaient en fait deux stratégies de déplacement lors d'une année de sécheresse : l'une qui consistait à aller, ou rester pour ceux qui y étaient déjà, sur les parcours qui avaient reçu les rares précipitations ; l'autre qui consistait à rester à proximité du point d'eau et à supporter le manque de disponibilité alimentaire sur les parcours en jouant sur l'état des dromadaires et la supplémentation alimentaire (figure 4). Il semble que deux visions de la conduite des dromadaires sur parcours se dessinent à travers ces stratégies, certains éleveurs favorisent l'alimentation alors que d'autres favorisent l'abreuvement.

De la même manière, l'analyse multivariée des variables de déplacement en 1996 (LOCTSS96, LOCTSH95 et DEP96) (tableau II), année favorable pour les parcours, a déterminé trois classes :

- la première classe comptait 67 troupeaux, dont la localisation en saison sèche était toujours la même chaque année. Ils ont effectué un déplacement de moins de $500 \mathrm{~km}$ vers l'intérieur, au sud de Laâyoune ;

- la deuxième classe comptait 58 troupeaux qui ne se sont pas déplacés cette année-là et dont la localisation en saison sèche était toujours la même chaque année ;

- la troisième classe regroupait 42 troupeaux dont la localisation en saison sèche variait chaque année. Ils ont effectué un déplacement de plus de $500 \mathrm{~km}$ vers l'intérieur des terres ou vers la côte océanique, au sud de Laâyoune.

Ces trois classes représentaient donc trois stratégies de déplacement lors d'une année favorable, au cours de laquelle les précipitations étaient relativement abondantes et bien réparties sur les parcours. Ces trois stratégies consistaient à se déplacer sur de grandes distances, se déplacer sur des distances plus courtes, ou enfin ne pas se déplacer et rester à proximité du point d'eau sur les parcours de saison sèche tout en profitant de l'effet des précipitations sur les parcours (figure 5).

Le croisement des typologies des déplacements en 1995 et 1996 révèle une corrélation entre les classes de chaque typologie. L'analyse du tableau croisé permet de déterminer quatre grandes classes de stratégies générales de déplacement en fonction des conditions climatiques et de la disponibilité alimentaire :

- la première stratégie a été adoptée par 71 troupeaux qui se déplaçaient systématiquement, quelles qu'aient été les conditions de milieu, souvent sur des distances supérieures à $500 \mathrm{~km}$, pour trouver les parcours offrant l'alimentation la plus riche en qualité et en quantité ;

- la deuxième stratégie de déplacement concernait 24 troupeaux qui effectuaient un grand déplacement de plus de $500 \mathrm{~km}$ lorsque les conditions bioclimatiques étaient défavorables, également pour trouver les parcours offrant les meilleures disponibilités alimentaires ;

- la troisième stratégie a été adoptée par 38 troupeaux qui ne se déplaçaient que si les conditions de milieu étaient favorables. Le déplacement pouvait alors être supérieur ou inférieur à $500 \mathrm{~km}$. Ces éleveurs favorisaient l'abreuvement de leurs dromadaires et ne se déplaçaient que s'ils étaient sûrs de pouvoir trouver des parcours de bonne qualité avec une alimentation riche en eau pour pouvoir s'éloigner du point d'eau, donc uniquement lors des années humides ;

- enfin, 34 troupeaux ne se déplaçaient jamais, quelles qu'aient été les conditions bioclimatiques, mais restaient localisés autour du même point d'eau. Leur mode d'élevage n'était absolument pas nomade et privilégiait l'abreuvement des dromadaires au détriment de leur alimentation, surtout en année de sécheresse. 


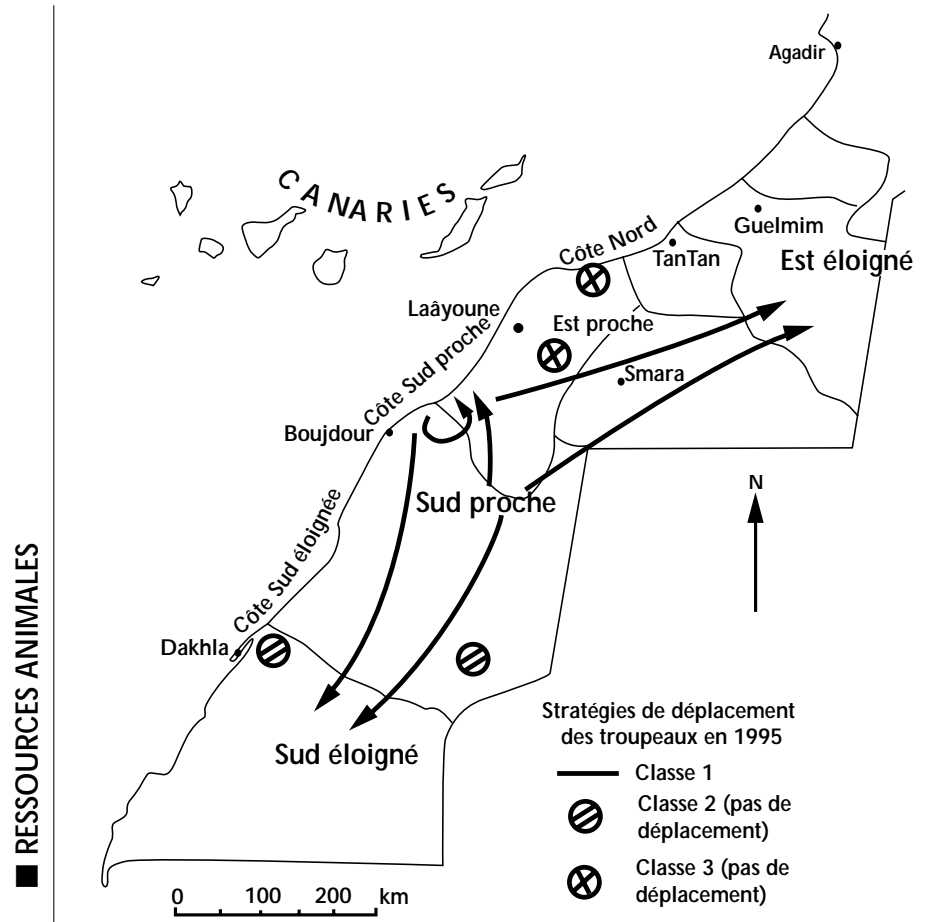

Figure 4 : typologie des déplacements de troupeaux en 1995.

Cette typologie des stratégies de déplacement en fonction des conditions bioclimatiques a donc révélé des différences de priorité selon les éleveurs pour la conduite de l'alimentation de leurs dromadaires. De plus, ces stratégies n'étaient reliées à aucun autre caractère du troupeau, comme la taille par exemple.

\section{Typologie des systèmes de production basée sur les pratiques d'élevage}

Les pratiques d'élevage considérées (tableau IV) étaient essentiellement les pratiques de mise bas (SURVMBAS, COLOSTAVOL, AIDECOL et TRAITEMIN), de sevrage (SEVMIN et MODESEVRAGE), de supplémentation (SUPPLEM) et les pratiques thérapeutiques (PLTESTOX, TRAITEMENT, DEPAREXT et VACCIN). En ce qui concerne les pratiques de mise bas, 87 p. 100 des éleveurs laissaient le nouveau-né prendre le colostrum à volonté si celui-ci était suffisamment fort, mais 93 p. 100 des éleveurs limitaient la prise colostrale par le nouveau-né si celui-ci était faible. Les pratiques de supplémentation, déjà décrites, présentaient peu de variabilité puisque seulement 4 p. 100 des éleveurs ne supplémentaient jamais leurs dromadaires. Les pratiques thérapeutiques et prophylactiques étaient peu variables d'un éleveur à l'autre. En effet 2 p. 100 des éleveurs n'utilisaient que des traitements traditionnels, alors que les autres utilisaient des traitements modernes exclusivement ou en complément des traitements traditionnels, 97 p. 100 des éleveurs effectuaient un déparasitage externe des animaux infestés, 97 p. 100 des éleveurs ne pratiquaient pas de mise à l'écart des animaux introduits dans le troupeau et 90 p.100 des éleveurs ne vaccinaient pas leurs animaux contre la variole cameline. Enfin, le marquage des animaux était réalisé généralement à 1 an (78 p. 100 des éleveurs) lorsque le jeune était sevré ou en passe de l'être.

Cette homogénéité des pratiques était certainement à l'origine des difficultés rencontrées pour les typer et du déséquilibre des classes obtenues. En effet les axes déterminés par l'analyse en compo-

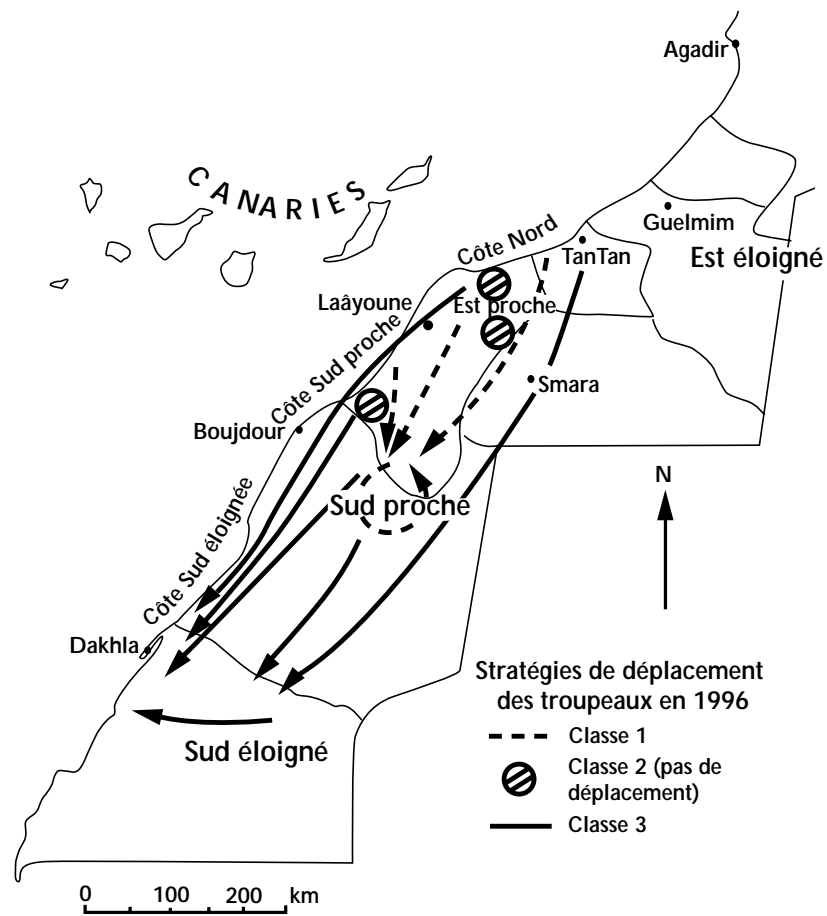

Figure 5 : typologie des déplacements de troupeaux en 1996.

santes multiples ont un faible pourcentage d'inertie associée, et aucun ne se détache nettement. Aucune variable ne semble être suffisamment discriminante. Cependant une classification ascendante hiérarchique, effectuée sur les six premiers facteurs (47 p. 100 de l'inertie du nuage), détermine trois classes, essentiellement caractérisées par l'âge minimum des jeunes au sevrage (SEVMIN) et au début de la traite (TRAITEMIN). Cette typologie des pratiques d'élevage (tableau VII) est constituée d'une classe principale et de deux classes marginales :

- une classe très importante d'éleveurs $(n=119)$ qui sevraient en général le jeune au moins à 1 an et débutaient la traite des femelles lorsque le jeune avait 4 ou 5 mois. Classique (6), cette stratégie permettait au jeune de profiter suffisamment du lait et limitait l'impact de la lactation sur les femelles ;

- une classe d'éleveurs $(n=47)$ qui effectuaient un sevrage précoce et traitaient rapidement les femelles après la mise bas. Ils exploitaient les capacités reproductrices de leurs chamelles en diminuant l'intervalle entre mises bas. Cette stratégie était défavorable aux jeunes, qui profitaient peu du lait, et aux femelles fréquemment en gestation ;

- une petite classe d'éleveurs $(n=10)$ qui n'avaient pas de réelle stratégie d'exploitation du troupeau : un élevage « naturel », pratiquement autogéré par les animaux en ce qui concernait leur reproduction. Cette stratégie était certainement bénéfique pour les jeunes dromadaires qui profitaient longtemps et à volonté du lait maternel, mais peut-être moins pour les femelles dont les intervalles entre mises bas étaient longs.

Le croisement des quatre typologies indique que seules les typologies d'organisation des éleveurs et de structure des troupeaux étaient corrélées. Les différentes stratégies de déplacement et de pratique d'élevage n'étaient pas corrélées entre elles ni avec les deux typologies précédentes. De même, aucune classe de ces différentes typologies n'était corrélée à une tribu particulière, malgré la prépondérance des Zerguiyines dans la population interrogée. 


\section{Tableau VII}

Description des classes de la typologie des pratiques d'élevage par le pourcentage des modalités des variables dans chaque classe

\begin{tabular}{|c|c|c|c|c|}
\hline \multirow[b]{2}{*}{ Variable } & \multirow[b]{2}{*}{ Mod. } & \multicolumn{3}{|c|}{ Classes de la typologie } \\
\hline & & 1 & 2 & 3 \\
\hline \multirow{2}{*}{$\begin{array}{l}\text { Nb. de } \\
\text { troupeaux }\end{array}$} & & 10 & 119 & 47 \\
\hline & & & $\%$ & \\
\hline \multirow[t]{4}{*}{ SURVMBAS } & 1 & 20 & 24 & 45 \\
\hline & 2 & 20 & 25 & 4 \\
\hline & 3 & 10 & 45 & 45 \\
\hline & 4 & 50 & 6 & 6 \\
\hline \multirow[t]{2}{*}{ COLOSTAVOL } & 1 & 80 & 89 & 83 \\
\hline & 2 & 20 & 11 & 17 \\
\hline \multirow[t]{2}{*}{ AIDECOL } & 1 & 10 & 8 & 2 \\
\hline & 2 & 90 & 92 & 98 \\
\hline \multirow[t]{4}{*}{ TRAITEM IN } & 1 & - & - & 51 \\
\hline & 2 & 20 & 60 & 23 \\
\hline & 3 & 40 & 38 & 23 \\
\hline & 4 & 40 & 2 & 2 \\
\hline \multirow[t]{3}{*}{ SEVMIN } & 1 & 10 & 8 & 30 \\
\hline & 2 & 90 & 81 & 66 \\
\hline & 3 & - & 11 & 4 \\
\hline \multirow[t]{3}{*}{ MODESEVRAGE } & 1 & 20 & 32 & 64 \\
\hline & 2 & 20 & 56 & 36 \\
\hline & 3 & 60 & 12 & - \\
\hline \multirow[t]{2}{*}{ PLTESTO X } & 1 & 40 & 33 & 66 \\
\hline & 2 & 60 & 67 & 34 \\
\hline \multirow[t]{2}{*}{ SUPPLEM } & 1 & 100 & 100 & 85 \\
\hline & 2 & - & - & 15 \\
\hline \multirow[t]{3}{*}{ TRAITEM EN T } & 1 & 70 & 72 & 87 \\
\hline & 2 & 30 & 24 & 13 \\
\hline & 3 & - & 4 & - \\
\hline \multirow[t]{2}{*}{ DEPAREXT } & 1 & 40 & 100 & 100 \\
\hline & 2 & 60 & - & - \\
\hline \multirow[t]{2}{*}{ VACCIN } & 1 & - & 13 & 4 \\
\hline & 2 & 100 & 87 & 96 \\
\hline
\end{tabular}

\section{DISCUSSION}

L'élevage camelin dans les provinces du Sud au Maroc est fortement influencé par le conflit passé et la forte intervention actuelle de l'Etat. Il est en cours de mutation et cette enquête ne prétend en aucun cas décrire cet élevage à une échelle régionale où la situation est différente (4). Les données bibliographiques concernant les systèmes de production camelin dans les provinces du Sud sont rares et les études n'ont été réalisées que sur quelques troupeaux $(1,5,12)$. Lors de cette enquête, les informations obtenues concernaient la moitié du cheptel camelin recensé dans la province de Laâyoune et ont permis de préciser les structures d'organisation humaine, des troupeaux, les stratégies de déplacement et de pratiques d'élevage. Une réserve est cependant à formuler en ce qui concerne la tribu des éleveurs interrogés, en grande majorité de la tribu des Zerguiyines. Il est certain que ce biais était inévitable par la présence d'un guide Zerguiyine lors des sorties sur le terrain. Cependant, d'après les experts locaux, leurs pratiques ne diffèrent pas de celles des autres groupes tribaux.
La stratégie d'analyse adoptée a permis d'exploiter au mieux les informations relevées et d'analyser plus finement les divers aspects de l'élevage camelin. Seules les typologies d'organisation humaine et de structure des troupeaux étaient corrélées. Ce résultat était attendu puisque la variable d'effectif (EFFECTIF) introduite dans l'analyse multivariée de l'organisation des éleveurs en variable illustrative était fortement corrélée aux classes déterminées. Cette typologie des éleveurs selon leur organisation, corrélée à la taille du troupeau, est la plus nette, statistiquement et par rapport à la réalité de l'élevage camelin sur le terrain. Les différentes stratégies de déplacement et de pratiques se répartissent dans toutes ces classes d'éleveurs. Les stratégies de pratiques ont été insuffisamment précisées, du fait notamment de la brièveté des entretiens et du niveau d'enquête.

L'analyse de l'organisation humaine autour de l'élevage camelin fait apparaître la diversité des objectifs des éleveurs et la mutation en cours de l'élevage camelin. Les éleveurs ont su s'adapter et profiter du progrès des moyens techniques, et la généralisation de l'automobile en est la preuve. Leurs conditions de vie ont également évolué depuis 20 ans puisqu'ils possèdent presque tous un lieu d'habitation fixe en ville, témoin de la politique marocaine de sédentarisation de la population. Cette généralisation a certainement modifié les pratiques des éleveurs, leur organisation et leurs rapports avec l'élevage en raccourcissant les distances et les délais de réaction. En effet les éleveurs peuvent rapidement connaître les événements qui ont lieu dans leurs troupeaux et réagir vite, mais ils peuvent aussi se permettre d'exercer d'autres activités en confiant la gestion journalière du troupeau à un berger, ou, à un niveau supérieur, la gestion de l'élevage à un contremaître. Cependant l'emploi d'un contremaître, véritable chef d'un secteur d'activité parmi l'ensemble des affaires du propriétaire, reste rare et ne concerne que les éleveurs riches possédant un grand nombre de dromadaires répartis en plusieurs troupeaux. En revanche, l'emploi d'un berger pour effectuer les tâches journalières de la conduite du troupeau semble plus fréquent. Il se généralise lorsque l'effectif dépasse 50 dromadaires, et permet de décharger le propriétaire d'une partie du travail. Mais le propriétaire reste seul maitre de la gestion de son troupeau et le berger, malgré son rôle capital, n'est souvent qu'un ouvrier temporaire exécutant. A ce titre les bergers des provinces d'Ouarzazate et d'Essaouira sont appréciés pour leur savoir-faire. Si le propriétaire semble donc le seul interlocuteur permettant d'accéder aux informations rétrospectives concernant le troupeau, le berger est l'interlocuteur privilégié pour obtenir des informations journalières plus fines concernant les individus du troupeau. Finalement il semble que ce soit plus l'organisation, la capacité financière et la ou les activités du propriétaire qui conditionnent l'encadrement humain de l'élevage et la taille des troupeaux, que l'inverse. La taille des troupeaux influence un certain nombre de pratiques. Mais si l'augmentation du nombre d'individus dans le troupeau entrâne une augmentation des difficultés, des moyens humains et financiers à mettre en œuvre, elle offre également des avantages. Les animaux rassemblés en lots bénéficient d'une conduite plus adaptée à chacun de leur statut physiologique. Le nombre de femelles traites est inférieur. Les capacités financières des gros propriétaires leur permettent d'acheter des aliments ou des médicaments sans avoir recours à un déstockage d'animaux, contrairement aux éleveurs plus modestes. Malgré tout, il existe des limites techniques, et il sera plus facile pour un éleveur modeste d'apporter les aliments nécessaires à ses animaux que pour un éleveur important, même si le parc automobile de certains éleveurs compte plusieurs dizaines de véhicules.

En ce qui concerne les stratégies de déplacement, même si le nomadisme n'est pas une pratique totalement généralisée dans la pro- 
vince de Laâyoune, puisque 19 p. 100 des éleveurs restent à proximité d'un point d'eau et ne se déplacent jamais, il concerne malgré tout une majorité d'éleveurs. Cependant cette pratique nomade est en voie de transformation. En effet, les éleveurs ont tendance à revenir passer la saison sèche au même endroit chaque année, en général à proximité de la ville dans laquelle ils habitent. Soixante-dix pour cent d'entre eux ont adopté cette pratique. Le nomadisme est toutefois réel en saison humide. Ces stratégies de déplacement sont variables, certains éleveurs se déplacent chaque année souvent sur de grandes distances, d'autres conditionnent leurs déplacements aux conditions climatiques et deux stratégies sont adoptées en cas de baisse de la disponibilité alimentaire sur les parcours :

- l'une qui consiste à rester au point d'eau, limiter l'impact du déficit alimentaire des parcours par la supplémentation et ne se déplacer que lors des années favorables ;

- l'autre qui consiste à se déplacer sur des parcours, repérés par prospection automobile, qui ont reçu des précipitations et à ne pas se déplacer lors des années favorables, les disponibilités alimentaires des parcours proches des points d'eau étant suffisantes.

Ces stratégies de déplacement ne correspondent pas à un type d'élevage précis, mais sont adoptées indifféremment par tous les éleveurs quelle que soit leur importance. Cependant les petits troupeaux se regroupent fréquemment s'ils doivent effectuer de grands déplacements. L'analyse de ces stratégies de déplacement permet de déterminer deux visions de la conduite alimentaire des dromadaires, l'une qui favorise l'utilisation des parcours et l'autre qui privilégie l'utilisation des points d'eau, sans que ces stratégies ne soient reliées à aucun autre caractère de structure du troupeau ou de pratiques d'élevage.

\section{- CONCLUSION}

D'après la quantité d'informations obtenues, le nombre d'interlocuteurs et la variété des élevages rencontrés, la majorité des situations épidémiologiques semble avoir été abordée. La typologie des systèmes de production camelin dans les provinces du Sud la plus pertinente est fondée sur l'organisation des hommes autour du troupeau. Elle fait apparaître quatre classes d'éleveurs, des petits éleveurs bergers, des éleveurs de moyenne importance très attentifs à leur élevage, des gros éleveurs qui ont une autre activité et qui emploient du personnel pour s'occuper de leur élevage et enfin de très gros éleveurs, peu nombreux, mais qui ont un poids important par la taille de leur cheptel, le personnel qu'ils emploient et par leur rôle d'éleveurs multiplicateurs. Mise en relation avec la mortalité des jeunes dromadaires avant sevrage, étudiée également lors de cette enquête (18), cette typologie a permis d'émettre des hypothèses de facteurs de risque, comme l'existence de toxiques alimentaires, les pratiques de traite et de sevrage, le niveau de présence du propriétaire, le confiage de la gestion du troupeau et le degré d'encadrement humain des jeunes dromadaires. De plus, cette clef de typologie, facilement identifiable sur le terrain, pourra servir de base d'échantillonnage pour des protocoles de suivis individuels ultérieurs qui permettront de préciser les différentes pratiques d'élevage encore mal déterminées et de confirmer les hypothèses de facteurs de risque de la mortalité des jeunes. La levée de cette contrainte majeure de l'élevage camelin dans les provinces sahariennes est une condition préalable au développement de cette activité d'autant plus importante que le marché intérieur et extérieur, représenté notamment par les Iles Canaries, en produits camelins est loin d'être satisfait.

\section{Remerciements}

Nous tenons à remercier les Services vétérinaires et de Productions animales des provinces de Laâyoune et Dakhla, ainsi que le Ministère de l'Agriculture et de la Mise en valeur agricole du Maroc, qui nous ont donné toute facilité pour mettre en œuvre cette enquête sur le terrain.

\section{BIBLIO GRAPHIE}

1. BAHIYA M., 1995. Gestion et productivité des élevages de dromadaires dans la région d' $\mathrm{O}$ ued Eddaheb. Mémoire $3^{\mathrm{e}}$ cycle Agron., Ecole natl. Agric., Meknès, Maroc, 178 p.

2. BIROUK A., LEWALLE J., TAZI M., 1991. Le patrimoine végétal des provinces sahariennes du Maroc. Rabat, Maroc, Actes Ed., 76 p. (coll. Documents scientifiques et techniques)

3. BO N NET P., 1995. Mission de suivi et d'identification d'opérations de recherche dans l'espèce cameline en milieu saharien. Maisons-Alfort, France, CIRAD-EMVT, $93 \mathrm{p}$.

4. BONTE P., 1995. Mauritanie : nomades en transition. Hist. Dév., 30 : 10-13.

5. CHRIQUI A., 1988. Conduite de l'élevage dans le Sud marocain (bilan et possibilités d'amélioration). Thèse doct. vét., Inst. Agron. vét. Hassan II, Rabat, Maroc, 155 p.

6. DIAGANA D., 1977. Contribution à l'étude de l'élevage du dromadaire en Mauritanie. Thèse doct. vét., Ecole Inter Etats Sci. Méd. vét., Dakar, Sénégal, 148 p.

7. EPI INFO, vers. 6, 1994. Center for disease control and prevention. Geneva, Switzerland, OMS.

8. FASSI FEHRI M.F.A., 1988. L'élevage camelin : situation actuelle et perspectives. In : ler Congr. natl vét., Laâyoune, Maroc, 18-20 mars 1988, Assoc. natl. des Vétérinaires du Maroc.

9. FAYE B., BRO CHARD, 1986. Enquête écopathologique continue : 7. Approche épidémiologique des mammites post-partum chez la vache laitière : étude descriptive et typologie des élevages. Ann. Rech. vét., 17 : 297-311.

10. FAYE B., LEFEVRE P.C., LANCELOT R., QUIRIN R., 1994. Ecopathologie animale; méthodologie, applications en milieu tropical. Maisons-Alfort, France, CIRAD, 119 p. (coll. Du labo au terrain)

11. FENELON J.P., 1981. Q u'est-ce que l'analyse des données? Paris, France, Lefonen Ed., $311 \mathrm{p}$.

12. JABRA A., 1989. L'élevage camelin dans la province de Guelmim : situation actuelle et perspectives. Thèse doct. vét., Inst. Agron. vét. Hassan II, Rabat, Maroc, 126 p.

13. Rapport, Direction provinciale de l'agriculture de Laâyoune, 1995. Laâyoune, Maroc, Direction provinciale de l'agriculture de Laâyoune.

14. Rapport, Institut national de la recherche agronomique, 1996. Laâyoune, Maroc, Institut national de la recherche agronomique.

15. Rapport, Ministère de l'agriculture et de la réforme agraire, 1986. Rabat, Maroc, Ministère de l'agriculture et de la réforme agraire.

16. Rapport, Station météorologique de Laâyoune, 1993. Laâyoune, M aroc, Station météorologique de Laâyoune.

17. SPAD, vers. 3.01, 1996. Logiciel d'analyse des données. St Mandé, France, CISIA.

18. MICHEL J.F., 1996. Enquête sur les systèmes de production camelins dans la province de Laâyoune ( $M$ aroc) : réalisation d'une typologie. DESS Prod. Anim. en régions chaudes. Montpellier, France, CIRADEMVT, $118 \mathrm{p}$.

Reçu le 5.7.97, accepté le 10.3.98 


\section{Summary}

Michel J.F., Bengoumi M., Bonnet $P_{.}$, Hidane K., Zro K., Faye B. Typology of dromedary production systems in Laayoune province, Morocco

In the Laayoune province of Morocco young dromedary mortality is high before weaning, while no etiology has been clearly identified. A farmers' and farmers' practice typology was established from a retrospective cross survey in Laayoune province in order to set up a sampling base to be used in selecting herds for future monitoring. By the end of the survey, 170 farmers had been consulted (10\% of all registered farmers), representing 12,000 dromedaries $(44 \%$ of dromedaries registered in Laayoune province). Four types of farmers' organization based on herd size, 4 types of moving strategies according to bioclimatic conditions and 3 types of farming practices were determined after a multivariate analysis was performed on data assembled by themes. These three typologies were not correlated. Most epidemiological situations seemed to have been covered when considering the amount of information obtained, the number of people surveyed and the variety of farm types. The most sensible typology of dromedary production systems in Laayoune province was based on men's organization around the herd. This typology, which takes young dromedary mortality before weaning into account, helped elaborate hypotheses on risk factors, such as toxic plants, milking and weaning practices, owner's attendance level, delegation of herd management and human supervision level of young dromedaries. In addition, this typology key, easily identifiable in the field, can be used as a sampling basis in future individual monitoring protocols. The protocols will help define more precisely the various farming practices and confirm hypotheses on risk factors in young mortality.

Key words: Dromedary - Camelus dromedarius - Young animal - Risk - Mortality - Classification - Epidemiology Morocco.

\section{Resumen}

Michel J.F., Bengoumi M., Bonnet P., Hidane K., Zro K., Faye B. Tipología de los sistemas de producción de camélidos en la provincia de Laayoune, Marruecos

En la provincia de Laayoune, en Marruecos, la mortalidad de los dromedarios jóvenes antes del destete era importante, sin que se hubiese identificado ninguna etiología evidente. Se realizó una tipología de los criadores y de sus prácticas, mediante encuesta transversal retrospectiva de los criadores de la provincia de Laayoune, esto con el fin de disponer de una base de muestras para elegir los hatos a seguir. Al cabo de la encuesta, se interrogaron 170 criadores, o sea $10 \%$ de los criadores en el censo, representando 12000 dromedarios, es decir $44 \%$ de los dromedarios en el censo de la provincia de Laayoune. Un análisis multi variado de los datos agrupados por temas, permitió determinar 4 tipos de organizaciones de los criadores, en función del tamaño del hato, 4 tipos de estrategias de desplazamiento, según las condiciones bioclimáticas y 3 tipos de prácticas de manejo. Estas tres tipologías no estuvieron correlacionadas. Según la cantidad de información obtenida, el número de interlocutores y la variedad de los establecimientos encontrados, parece haberse cubierto la mayoría de las situaciones epidemiolgógicas. La tipología más pertinente de los sistemas de producción de camélidos en la provincia de Laayoune, se basa en la organización de los hombres alrededor de un hato. La comparación de esta tipología con la mortalidad de los dromedarios jóvenes pre destete, permite emitir hipótesis sobre los factores de riesgo, como la existencia de tóxicos alimenticios, las prácticas de ordeño y de destete, el grado de presencia del propietario, la delegación del manejo del hato y el grado de cuidado humano de los jóvenes dromedarios. Además, esta tipología, fácilmente identificable en el campo, servirá como base de muestreo para los protocolos en seguimientos individuales ulteriores, los cuáles permitirán identificar las diferentes prácticas de manejo, aún mal conocidas y confirmar las hipótesis sobre los factores de riesgo de mortalidad de los jóvenes.

Palabras clave: Dromedario - Camelus dromedarius - Animal joven - Riesgo - Mortalidad - Clasificación - Epidemiología Marruecos. 